\title{
Frequent Premature Atrial Contractions as a Poor Prognostic Factor in Cryptogenic Stroke Patients with Concomitant Non-Sustained Atrial Tachycardia
}

\author{
Jung-Joon Cha' ${ }^{1}$, Kyung-Yul Lee ${ }^{2}$, Hyemoon Chung ${ }^{3}$, In-Soo Kim², Eui-Young Choi ${ }^{4}$, \\ Pil-Ki Min ${ }^{4}$, Young Won Yoon ${ }^{4}$, Byoung Kwon Lee ${ }^{4}$, Bum-Kee Hong ${ }^{4}$, \\ Se-Joong Rim ${ }^{4}$, Hyuck Moon Kwon ${ }^{4}$, and Jong-Youn Kim ${ }^{4}$ \\ ${ }^{1}$ Division of Cardiology, Cardiovascular Center, Korea University Anam Hospital, Korea University College of Medicine, Seoul; \\ ${ }^{2}$ Department of Neurology, Gangnam Severance Hospital, Yonsei University College of Medicine, Seoul; \\ ${ }^{3}$ Department of Cardiology, Department of Internal Medicine, Kyung Hee University, Seoul; \\ ${ }^{4}$ Division of Cardiology, Gangnam Severance Hospital, Yonsei University College of Medicine, Seoul, Korea.
}

In cryptogenic stroke patients, early detection of new-onset atrial fibrillation (AF) and recurrent stroke is required to prevent poor clinical outcomes. Therefore, we investigated the predictors of new-onset AF and recurrent stroke in cryptogenic stroke patients without previously diagnosed AF. In total, 390 patients who were diagnosed with stroke and non-sustained atrial tachycardia (NSAT) on 24-hour Holter monitoring were followed up to assess new-onset AF and recurrent stroke. The 5-year event-free survival as well as the predictors of recurrent stroke or new-onset AF were investigated. Based on receiver operating characteristic analysis, frequent premature atrial contractions (PACs) were defined as PACs $>44$ beats/day. The median follow-up period was 35 months. The composite event rate was $11.5 \%$. In Kaplan-Meier analysis, the 5 -year cumulative incidence of composite events was higher in cryptogenic stroke patients with frequent PACs than in those without frequent PACs. Multivariate analysis revealed that current smoking, increased left atrial volume index, and frequent PACs were poor prognostic predictors of composite event, and frequent PACs were an independent poor prognostic factor of new-onset AF in cryptogenic stroke patients. Therefore, frequent PACs might be associated with poor clinical outcomes (new-onset AF and recurrent stroke) in cryptogenic stroke patients with concomitant NSAT.

Key Words: Cryptogenic stroke, premature atrial contraction, clinical outcome, atrial fibrillation, recurrent stroke

Stroke is a clinically heterogeneous entity. Based on the heart disease and stroke statistics from the American Heart Association, ischemic stroke, intracerebral hemorrhage, and subarachnoid hemorrhages account for $87 \%, 10 \%$, and $3 \%$ of all

\footnotetext{
Received: September 11, 2020 Revised: September 24, 2020

Accepted: September 28, 2020

Corresponding author: Jong-Youn Kim, MD, PhD, Division of Cardiology, Gangnam Severance Hospital, Yonsei University College of Medicine, 211 Eonju-ro, Gangnam-gu, Seoul 06273, Korea

Tel: 82-2-2019-3310, Fax: 82-2-3463-3882, E-mail: jykim0706@yuhs.ac

-The authors have no potential conflicts of interest to disclose.

(C) Copyright: Yonsei University College of Medicine 2020

This is an Open Access article distributed under the terms of the Creative Commons Attribution Non-Commercial License (https://creativecommons.org/licenses/ by-nc/4.0) which permits unrestricted non-commercial use, distribution, and reproduction in any medium, provided the original work is properly cited.
}

strokes, respectively. ${ }^{1}$ Approximately a quarter of all ischemic strokes are cryptogenic. ${ }^{2}$ Moreover, patients with atrial fibrillation (AF) had severe clinical presentation or sequelae of cerebral infarction compared to those without AF; hence, early detection of AF significantly improves patient prognosis and reduces the overall healthcare costs. ${ }^{3}$ Previous studies have reported that premature atrial contractions (PACs) and non-sustained atrial tachycardia (NSAT) observed on 24-hour Holter monitoring may predict undiagnosed $\mathrm{AF}^{4,5}$ We investigated whether frequent PACs predicted new-onset $\mathrm{AF}$ and recurrent stroke in cryptogenic stroke patients without previously diagnosed AF.

A total of 390 patients with concomitant NSAT from 2091 patients who were diagnosed with acute ischemic stroke without intracardiac thrombi were included in the study between Janu- 
ary 2009 and December 2012. All of the patients were not diagnosed with AF before the first episode of acute ischemic stroke. The diagnosis of stroke was based on the International Classification of Diseases, Ninth Revision. For neurological evaluation, patients underwent magnetic resonance imaging or computed tomography. Brain magnetic resonance imaging and angiography were performed to exclude patients with atherosclerotic or cardioembolic strokes and structural heart diseases. In addition, patients with intracardiac thrombi were excluded by transesophageal echocardiography. Therefore, in the Trial of ORG 10172 in Acute Stroke Treatment (TOAST) classification, ${ }^{6}$ etiologies of the enrolled patients were small vessel disease and other determined and undetermined etiologies. NSAT was diagnosed by 24-hour Holter monitoring on current admission. NSAT was defined as $\geq 3$ consecutive PACs with a heart rate of $>100$ beats $/ \mathrm{min}$ for $<30 \mathrm{~s}$. PAC was defined based on the following electrocardiographic parameters: 1) a shortened RR interval ( $\geq 25 \%$ reduction in interval duration), 2) occurrence of a $\mathrm{P}$ wave, and 3) QRS width $<0.12 \mathrm{~s}$. Within 7 days of admission, all of the enrolled patients underwent transthoracic echocardiography, transesophageal echocardiography, and 24-hour Holter monitoring. The enrolled patients were admitted at three tertiary hospitals (Severance Hospital, Gangnam Severance Hospital, and Kyung Hee University Hospital). This study complied with the Declaration of Helsinki and was approved by the Institutional Review Board of the Gangnam Severance Hospital, Seoul, South Korea (3-2020-0260). Written informed consent was obtained from all patients.

The exclusion criteria were as follows: 1 ) history of stroke or transient ischemic attack; 2) history of a brain hemorrhage, brain tumor, and cerebrovascular malformations; 3) known tachyarrhythmias, including supraventricular tachyarrhythmia; 4) status post of a permanent pacemaker; 5) presence of atherosclerotic plaques involving the aorta or aortic arch and the carotid artery; 6) history of structural heart disease; 7) systolic dysfunction [ejection fraction (EF); <50\%]; and 8) history of neoplasm.

Patients were categorized into those without events $(n=345)$ and those with events $(n=45)$. Electronic medical records of the patients and relevant data were collected. After the first ischemic stroke event, the enrolled patients underwent standard ECG, 24-hour Holter monitoring, and echocardiography to identify cardioembolic sources of stroke. After that, antiplatelet agents (aspirin, clopidogrel, or triflusal) were prescribed. Recurrent stroke was defined as the occurrence of neurological symptoms associated with objective evidence of lesions on brain imaging. New-onset $\mathrm{AF}$ is a working diagnosis in patients without a history of AF, until the pattern of AF can be defined more precisely. After enrollment, all of the patients regularly visited the outpatient clinics after discharge to check for symptoms and undergo a standard 12-lead ECG recording every 3 months. Based on a standard 12-lead ECG recording or a single-lead ECG tracing lasting 30 seconds, heart rhythm with no discernible repeating $P$ waves and irregular RR intervals (when atrioventricular conduction is not impaired) was diagnosed as clinical AF.

According to the current American Society of Echocardiography guidelines, all of the patients underwent Doppler echocardiography to measure the following echo parameters: 1) peak early (E); 2) late diastolic mitral inflow velocities; 3) early diastolic mitral annulus peak velocity ( $\left.\mathrm{e}^{\prime}\right)$ from the septal aspect of the mitral annulus in the apical four-chamber view; 4) E/e' ratio, with an $\mathrm{E} / \mathrm{e}^{\prime}$ ratio of $>14$ defined as the presence of diastolic dysfunction ${ }^{7}$; 5) EF, which was calculated by Modified Simpson rule; 6) the left atrial (LA) anteroposterior diameter, which was measured in a parasternal long-axis view at end-systole to determine the left atrium size; 7) LA volume, which was measured by the ellipse method from the apical four-chamber and parasternal long-axis views at ventricular end-systole; and 8) LA volume index (LAVi), which was used to adjust LA volume regarding body surface area.

Categorical and continuous variables are presented as numbers (percentages) and mean \pm standard deviation, respectively. Baseline clinical characteristics were compared between patients with and without composite events using Student's ttest for continuous variables and the chi-square test for categorical variables. A $p$-value $<0.05$ was considered statistically significant. The Youden index based on receiver operating characteristic was used to determine the optimal cutoff value for composite events. Based on the area under the receiver operating characteristic curve value (0.602), PACs $>44$ beats/day were defined as frequent PACs (sensitivity: $71 \%$ and specificity: 52\%). Kaplan-Meier survival curves and log-rank test were used to compare the 5-year cumulative incidence of composite events between patients with and without frequent PACs. Univariate and multivariate Cox proportional hazards regression analyses were performed to determine independent poor predictors of composite events. Variables with a $p$-value $<0.20$ in univariate analysis or those considered relevant predictors of clinical events were included in the multivariate analysis. All statistical analyses were performed using the SPSS software ver. 25.0 (IBM Corp., Armonk, NY, USA).

The mean age of the 390 patients was 67 years. No statistically significant intergroup differences were noted in the baseline clinical characteristics, including the mean age $(p=0.110)$. However, compared to patients without clinical events, those with clinical events had a larger LA diameter (36.2 $\mathrm{mm}$ vs. 37.7 $\mathrm{mm}, p=0.044)$, LA volume (40.5 $\mathrm{mm}^{3}$ vs. $\left.46.1 \mathrm{~mm}^{3}, p=0.003\right)$, and LAVi (24.1 $\mathrm{mm}^{3} / \mathrm{m}^{2}$ vs. $\left.28 \mathrm{~mm}^{3} / \mathrm{m}^{2}, p=0.005\right)$ (Table 1). The median follow-up period was 35 months, and the composite event rate was $11.5 \%(45 / 390)$. With regard to individual clinical events, the rate of new-onset AF was $3.1 \%$, and that of stroke recurrence was $9 \%$. Kaplan-Meier analysis revealed that the 5 -year cumulative incidence of composite events (new-onset $\mathrm{AF}$ and recurrent stroke) was significantly higher among cryptogenic stroke patients with frequent PACs than among those 
Jung-Joon Cha, et al.

Table 1. Patient Characteristics

\begin{tabular}{|c|c|c|c|c|}
\hline & \multirow{2}{*}{ Total $(n=390)$} & \multicolumn{2}{|c|}{ Composite outcomes } & \multirow{2}{*}{$p$} \\
\hline & & No event ( $n=345)$ & Event $(n=45)$ & \\
\hline Age (yr) & $66.7 \pm 11.9$ & $66.4 \pm 11.7$ & $69.4 \pm 13.0$ & 0.110 \\
\hline Male sex & $219(56.2)$ & $195(56.5)$ & $24(53.3)$ & 0.806 \\
\hline Hypertension & $273(70.0)$ & $237(68.7)$ & $36(80.0)$ & 0.167 \\
\hline Diabetes & $107(27.4)$ & $99(28.7)$ & $8(17.8)$ & 0.172 \\
\hline Dyslipidemia & $160(41.0)$ & $143(41.4)$ & $17(37.8)$ & 0.757 \\
\hline Chronic kidney disease & $17(4.4)$ & $13(3.8)$ & $4(8.9)$ & 0.232 \\
\hline Vascular disease & $12(3.1)$ & $10(2.9)$ & $2(4.4)$ & 0.916 \\
\hline Coronary artery disease & $41(10.5)$ & $37(10.7)$ & $4(8.9)$ & 0.905 \\
\hline Cigarette smokers & $148(37.9)$ & $127(36.8)$ & $21(46.7)$ & 0.264 \\
\hline \multicolumn{5}{|l|}{ Echocardiographic parameters } \\
\hline LA diameter (mm) & $36.4 \pm 4.5$ & $36.2 \pm 4.4$ & $37.7 \pm 5.0$ & 0.044 \\
\hline LA volume $\left(\mathrm{mm}^{3}\right)$ & $41.2 \pm 11.8$ & $40.5 \pm 11.5$ & $46.1 \pm 12.9$ & 0.003 \\
\hline LA volume index $\left(\mathrm{mm}^{3} / \mathrm{m}^{2}\right)$ & $24.5 \pm 6.9$ & $24.1 \pm 6.5$ & $28.0 \pm 8.6$ & 0.005 \\
\hline $\operatorname{LVEF}(\%)$ & $66.6 \pm 8.8$ & $66.7 \pm 8.7$ & $66.0 \pm 9.1$ & 0.612 \\
\hline LV mass index $\left(\mathrm{g} / \mathrm{m}^{2}\right)$ & $106.1 \pm 28.9$ & $105.3 \pm 27.1$ & $112.4 \pm 40.2$ & 0.279 \\
\hline $\mathrm{E} / \mathrm{e}^{\prime}$ & $12.0 \pm 4.5$ & $11.8 \pm 4.1$ & $13.5 \pm 6.2$ & 0.082 \\
\hline PACs (n) & $541.0 \pm 2565.7$ & $537.4 \pm 2608.8$ & $568.5 \pm 2233.7$ & 0.939 \\
\hline Frequent PACs & $210(53.8)$ & $178(51.6)$ & 32 (71.1) & 0.017 \\
\hline \multicolumn{5}{|l|}{ Medication } \\
\hline Aspirin & 301 (77.2) & 264 (76.5) & $37(82.2)$ & 0.504 \\
\hline Triflusal & $203(52.1)$ & 182 (52.8) & $21(46.7)$ & 0.542 \\
\hline Clopidogrel & $30(7.7)$ & $29(8.4)$ & $1(2.2)$ & 0.243 \\
\hline
\end{tabular}

LA, left atrial; LV, left ventricular; LVEF, left ventricular ejection fraction; PAC, premature atrial contractions.

Data are presented as $n(\%)$.

without frequent PACs (15.2\% vs. 7.2\%, log-rank $p=0.003$ ) (Fig. 1A). In addition, individual Kaplan-Meier analysis revealed that compared to cryptogenic stroke patients without frequent PACs, those with frequent PACs had a higher rate for both newonset AF ( $4.8 \%$ vs. $1.1 \%$, log-rank $p=0.006$ ) (Fig. 1B) and recurrent stroke ( $11.4 \%$ vs. $6.1 \%$, log-rank $p=0.031$ ) (Fig. 1C).

Univariate and multivariate Cox proportional hazard regression analyses were performed to investigate the predictors of composite events in cryptogenic stroke patients (Table 2). Univariate analysis revealed that age [hazards ratio (HR): 1.03, 95\% confidence interval (CI): 1.00-1.06, $p=0.049$ ], LAVi (HR: 1.07, 95\% CI: 1.03-1.10, $p=0.002$ ), and frequent PACs (HR: 2.62, 95\% CI: $1.37-5.02, p=0.004)$ were poor predictors for composite event in cryptogenic stroke patients. Multivariate analysis revealed that cigarette smoking status (HR: 2.07, 95\% CI: 1.12-3.84, $p=$ 0.021), LAVi (HR: 1.05, 95\% CI: 1.01-1.08, $p=0.006$ ), and frequent PACs (HR: 2.30, 95\% CI: 1.16-4.56, $p=0.017$ ) were independent poor prognostic factors in cryptogenic stroke patients with concomitant NSAT after adjusting for confounders such as age, sex, $\mathrm{EF}$, diastolic dysfunction (E/e' >14), LAVi, hypertension, diabetes, coronary artery disease, chronic kidney disease, cigarette smoking status, and frequent PACs. Additionally, multivariate analysis for individual events revealed that compared with cryptogenic stroke patients without frequent PACs, those with frequent PACs had a higher risk of new-onset AF (HR: 5.34,
95\% CI: 1.10-25.85, $p=0.037)$ but not of stroke recurrence (HR: 1.99, 95\% CI: 0.94-4.20, $p=0.072$ ).

The main findings of this study are as follows: 1) frequent PACs are a poor prognostic factor for clinical outcomes in cryptogenic stroke patients and 2) frequent PACs are associated with new-onset AF in cryptogenic stroke patients. In context, we suggest that cryptogenic stroke patients with risk factors for poor predictors should be considered for close and prolonged follow-up with cardiac rhythm monitoring to improve clinical outcomes.

We found that frequent PACs were a poor prognostic factor for clinical outcomes in cryptogenic stroke patients with NSAT and no previously diagnosed AF. The definition of frequent PACs remains unclear. Previous studies have defined frequent PACs based on their study results, which represent a subjective interpretation $^{8-10}$; nevertheless, compared to cryptogenic stroke patients without frequent PACs, those with frequent PACs had poor clinical outcomes. ${ }^{8-10}$ Another study reported that the PAC burden detected by Holter monitoring might be associated with clinical outcomes. ${ }^{11}$

Although various studies have reported an association between frequent PACs and poor clinical outcome, the exact pathomechanism remains unclear. In our study, frequent PACs were associated with poor clinical outcomes in composite event and newonset AF, but not with recurrent stroke. Plausible reasons may 
be that PACs are known precursors of AF, which is a potential risk factor for stroke. ${ }^{5,12}$ Previous studies have reported an association between PACs and AF in cryptogenic stroke patients ${ }^{12-14}$

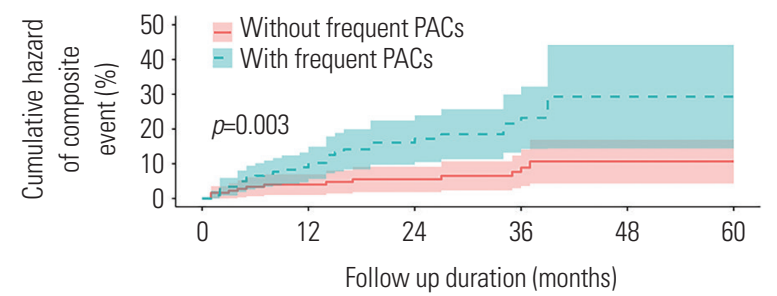

\begin{tabular}{|c|c|c|c|c|c|c|}
\hline \multicolumn{7}{|c|}{ Number at risk } \\
\hline Without frequent PACs & 180 & 149 & 107 & 84 & 42 & 39 \\
\hline With frequent PACs & 210 & 155 & 90 & 61 & 14 & 12 \\
\hline A & 0 & 12 & 24 & 36 & 48 & 60 \\
\hline
\end{tabular}
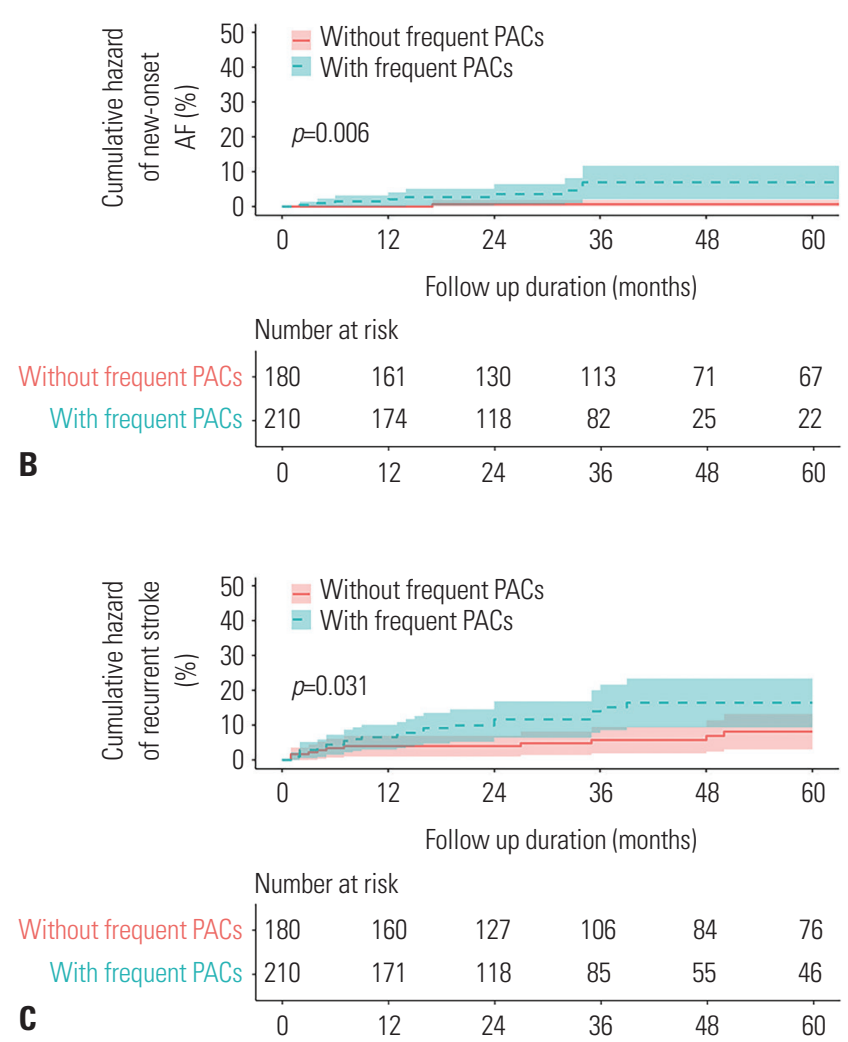

Fig. 1. Kaplan-Meier survival curves. (A) Composite clinical events. (B) Newonset $A F$. (C) Recurrent stroke. PAC, remature atrial contraction; $A F$, atrial fibrillation. and proved that frequent PACs or consecutive supraventricular extrasystoles, such as NSAT, are associated with a higher prevalence of AF. ${ }^{12-14}$ In our results, frequent PACs was defined as PACs $>44$ beats/day using the Youden index based on the receiver operating characteristic. The cut-off value used in our study was relatively small compared to that in the aforementioned study. However, despite the relatively small number of PAC, our results revealed that patients with frequent PACs had a potential risk of hidden AF. Therefore, intensive observation with a 12-lead ECG recording or the usage of a loop recorder for patients who have frequent PAC may offer better clinical outcomes by early detection of new-onset AF.

In addition, our results revealed that increased LAVi was a poor predictor of clinical outcomes. Previous studies have suggested LAVi as a risk factor for cardioembolic stroke. ${ }^{15,16}$ Also, Atrial stretch ${ }^{17}$ and atrial tissue alterations ${ }^{18}$ in an enlarged left atrium are associated with disturbances in impulse propagation in patients with $\mathrm{AF}$, suggesting that left atrium enlargement may increase the atrial ectopy rate and, consequently, the risk of stroke. ${ }^{19}$ In addition, the association between smoking status and recurrent stroke is statistically significant.

The smoking aggravates atherosclerosis and stroke occurrence by an increase of oxidative stress, endothelial dysfunction, and inflammation. ${ }^{20,21}$ In addition, smoking increases the risk of recurrent stroke. ${ }^{22}$ Furthermore, we suggest that patients with the abovementioned risk factors should be considered as having a high risk of poor clinical outcomes. Therefore, close monitoring of frequent PACs with 24-hour Holter monitoring or wearable devices for detecting arrhythmia may contribute to better clinical outcomes in cryptogenic stroke patients.

This study had some limitations. First, since this was a retrospective study based on data from three tertiary referral hospitals, the possibility of selection and referral bias cannot be excluded. Second, the study population was relatively small, and the study lacked follow-up 24-hour Holter monitoring. Third, the statistical model in the multivariate analysis may not have accurately analyzed unmeasured confounding factors, such as the patients' general health condition and the three-dimensional techniques used in transthoracic echocardiography. Therefore, frequent PACs are significantly associated with poor clinical outcomes in cryptogenic stroke patients with concomitant NSAT.

Table 2. Adjusted Multivariate Risk of Frequent PACs for Subsequent 5-Year Clinical Events

\begin{tabular}{|c|c|c|c|c|}
\hline & \multicolumn{2}{|c|}{ Event rate at 5 year (unadjusted) } & \multirow[b]{2}{*}{ Adjusted HR of frequent PACs $(95 \% \mathrm{CI})$} & \multirow[b]{2}{*}{$p$ value } \\
\hline & $\begin{array}{l}\text { Without frequent PACs } \\
\qquad(\mathrm{n}=180)\end{array}$ & $\begin{array}{l}\text { With frequent PACs } \\
\qquad(\mathrm{n}=210)\end{array}$ & & \\
\hline Composite events & $7.2 \%(13)$ & $15.2 \%(32)$ & $2.30(1.16-4.56)$ & 0.017 \\
\hline New-onset atrial fibrillation & $1.1 \%(2)$ & $4.8 \%(10)$ & $5.34(1.10-25.85)$ & 0.037 \\
\hline Recurrent stroke & $6.1 \%(11)$ & $11.4 \%(24)$ & $1.99(0.94-4.20)$ & 0.072 \\
\hline
\end{tabular}

$\mathrm{HR}$, hazard ratio; PAC, premature atrial contractions; $\mathrm{Cl}$, confidence interval.

Rates are Kaplan-Meier estimates at 5 year (number of events). P-value corresponds to the adjusted HR (95\% CI). All clinical endpoints were adjusted by age, sex. EF, diastolic dysfunction (E/e' >14), LAVi, hypertension, diabetes, coronary artery disease, chronic kidney disease, cigarette smoking status, and frequent PACs. 


\section{AUTHOR CONTRIBUTIONS}

Conceptualization: Kyung-Yul Lee and Jong-Youn Kim. Data curation: Kyung-Yul Lee, Hyemoon Chung, In-Soo Kim, Eui-Young Choi, Pil-Ki Min, Young Won Yoon, Byoung Kwon Lee, Bum-Kee Hong, Se-Joong Rim, Hyuck Moon Kwon, and Jong-Youn Kim. Formal analysis: Jung-Joon Cha and Jong-Youn Kim. Funding acquisition: Jong-Youn Kim. Investigation: Jung-Joon Cha, Kyung-Yul Lee, and Jong-Youn Kim. Methodology: Jung-Joon Cha and Jong-Youn Kim. Project administration: JongYoun Kim. Resources: Kyung-Yul Lee and Jong-Youn Kim. Software: Jung-Joon Cha. Supervision: Kyung-Yul Lee, Hyemoon Chung, In-Soo Kim, Eui-Young Choi, Pil-Ki Min, Young Won Yoon, Byoung Kwon Lee, Bum-Kee Hong, Se-Joong Rim, Hyuck Moon Kwon, and Jong-Youn Kim. Validation: Jung-Joon Cha, Kyung-Yul Lee, and Jong-Youn Kim. Visualization: Jung-Joon Cha. Writing-original draft: Jung-Joon Cha. Writing_review \& editing: Jong-Youn Kim. Approval of final manuscript: all authors.

\section{ORCID iDs}

$\begin{array}{ll}\text { Jung-Joon Cha } & \text { https://orcid.org/0000-0002-8299-1877 } \\ \text { Kyung-Yul Lee } & \text { https://orcid.org/0000-0001-5585-7739 } \\ \text { Hyemoon Chung } & \text { https://orcid.org/0000-0002-5615-6245 } \\ \text { In-Soo Kim } & \text { https://orcid.org/0000-0003-2801-5514 } \\ \text { Eui-Young Choi } & \text { https://orcid.org/0000-0003-3732-0190 } \\ \text { Pil-Ki Min } & \text { https://orcid.org/0000-0001-7033-7651 } \\ \text { Young Won Yoon } & \text { https://orcid.org/0000-0002-0907-0350 } \\ \text { Byoung Kwon Lee } & \text { https://orcid.org/0000-0001-9259-2776 } \\ \text { Bum-Kee Hong } & \text { https://orcid.org/0000-0002-6456-0184 } \\ \text { Se-Joong Rim } & \text { https://orcid.org/0000-0002-7631-5581 } \\ \text { Hyuck Moon Kwon } & \text { https://orcid.org/0000-0001-9901-5015 } \\ \text { Jong-Youn Kim } & \text { https://orcid.org/0000-0001-7040-8771 }\end{array}$

\section{REFERENCES}

1. Benjamin EJ, Muntner P, Alonso A, Bittencourt MS, Callaway CW, Carson AP, et al. Heart Disease and Stroke Statistics-2019 Update: A Report From the American Heart Association. Circulation 2019; 139(10):e56-e528.

2. Saver JL. Clinical Practice. Cryptogenic stroke. N Engl J Med 2016; 374:2065-74

3. Go AS, Hylek EM, Phillips KA, Chang Y, Henault LE, Selby JV, et al. Prevalence of diagnosed atrial fibrillation in adults: national implications for rhythm management and stroke prevention: the AnTicoagulation and Risk Factors in Atrial Fibrillation (ATRIA) Study. JAMA 2001;285:2370-5.

4. Stahrenberg R, Weber-Krüger M, Seegers J, Edelmann F, Lahno R, Haase B, et al. Enhanced detection of paroxysmal atrial fibrillation by early and prolonged continuous holter monitoring in patients with cerebral ischemia presenting in sinus rhythm. Stroke 2010;41: 2884-8.

5. Wallmann D, Tüller D, Kucher N, Fuhrer J, Arnold M, Delacretaz E. Frequent atrial premature contractions as a surrogate marker for paroxysmal atrial fibrillation in patients with acute ischaemic stroke. Heart 2003;89:1247-8.
6. Adams HP Jr, Bendixen BH, Kappelle LJ, Biller J, Love BB, Gordon $\mathrm{DL}$, et al. Classification of subtype of acute ischemic stroke. Definitions for use in a multicenter clinical trial. TOAST. Trial of Org 10172 in Acute Stroke Treatment. Stroke 1993;24:35-41.

7. Vinther KH, Tveskov C, Möller S, Auscher S, Osmanagic A, Egstrup K. Excessive premature atrial complexes and the risk of recurrent stroke or death in an ischemic stroke population. J Stroke Cerebrovasc Dis 2017;26:1163-70.

8. Binici Z, Intzilakis T, Nielsen OW, Køber L, Sajadieh A. Excessive supraventricular ectopic activity and increased risk of atrial fibrillation and stroke. Circulation 2010;121:1904-11.

9. Larsen BS, Kumarathurai P, Falkenberg J, Nielsen OW, Sajadieh A. Excessive atrial ectopy and short atrial runs increase the risk of stroke beyond incident atrial fibrillation. J Am Coll Cardiol 2015; 66:232-41.

10. Caballero L, Kou S, Dulgheru R, Gonjilashvili N, Athanassopoulos GD, Barone D, et al. Echocardiographic reference ranges for normal cardiac Doppler data: results from the NORRE Study. Eur Heart J Cardiovasc Imaging 2015;16:1031-41.

11. Inohara T, Kohsaka S, Okamura T, Watanabe M, Nakamura Y, Higashiyama A, et al. Long-term outcome of healthy participants with atrial premature complex: a 15-year follow-up of the NIPPON DATA 90 cohort. PLoS One 2013;8:e80853.

12. Kochhäuser S, Dechering DG, Dittrich R, Reinke F, Ritter MA, Ramtin S, et al. Supraventricular premature beats and short atrial runs predict atrial fibrillation in continuously monitored patients with cryptogenic stroke. Stroke 2014;45:884-6.

13. Chong BH, Pong V, Lam KF, Liu S, Zuo ML, Lau YF, et al. Frequent premature atrial complexes predict new occurrence of atrial fibrillation and adverse cardiovascular events. Europace 2012;14:942-7.

14. Gladstone DJ, Dorian P, Spring M, Panzov V, Mamdani M, Healey JS, et al. Atrial premature beats predict atrial fibrillation in cryptogenic stroke: results from the EMBRACE trial. Stroke 2015;46:93641.

15. Di Tullio MR, Sacco RL, Sciacca RR, Homma S. Left atrial size and the risk of ischemic stroke in an ethnically mixed population. Stroke 1999;30:2019-24.

16. Fatema K, Bailey KR, Petty GW, Meissner I, Osranek M, Alsaileek AA, et al. Increased left atrial volume index: potent biomarker for first-ever ischemic stroke. Mayo Clin Proc 2008;83:1107-15.

17. Satoh T, Zipes DP. Unequal atrial stretch in dogs increases dispersion of refractoriness conducive to developing atrial fibrillation. J Cardiovasc Electrophysiol 1996;7:833-42.

18. Keren G, Etzion T, Sherez J, Zelcer AA, Megidish R, Miller HI, et al. Atrial fibrillation and atrial enlargement in patients with mitral stenosis. Am Heart J 1987;114:1146-55.

19. Marcus GM, Dewland TA. Premature atrial contractions: a wolf in Sheep's clothing? J Am Coll Cardiol 2015;66:242-4.

20. Wolf PA, D’Agostino RB, Kannel WB, Bonita R, Belanger AJ. Cigarette smoking as a risk factor for stroke. The Framingham Study. JAMA 1988;259:1025-9.

21. Ambrose JA, Barua RS. The pathophysiology of cigarette smoking and cardiovascular disease: an update. J Am Coll Cardiol 2004;43: 1731-7.

22. Chen J, Li S, Zheng K, Wang H, Xie Y, Xu P, et al. Impact of Smoking Status on Stroke Recurrence. J Am Heart Assoc 2019;8:e011696. 